\title{
Effect of animal-sourced bioactive peptides on the in vitro development of mouse preantral follicles
}

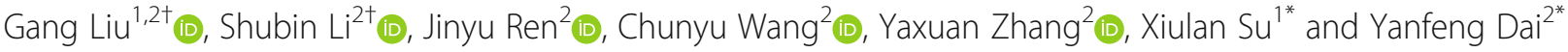

\begin{abstract}
The aim of this study was to investigate the effect of bioactive peptides (BAPT) from animal sources on the development of mouse preantral follicles in vitro. Preantral follicles were isolated and randomly divided into the following groups: an untreated group (control) and three groups supplemented with 20, 40 and $60 \mu \mathrm{g} / \mathrm{mL}$ BAPT, respectively. After establishing the in vitro follicle culture, the gene expression levels and hormone levels were quantified. After in vitro maturation, the developmental rates, reactive oxygen species (ROS) production levels and mitochondrial distributions of MII oocytes were investigated, followed by the analyses of embryonic developmental rates after in vitro fertilization.

The results showed that BAPT promoted the growth of mouse preantral follicles. Notably, after $14 \mathrm{~d}$ of in vitro culture, the levels of $17 \beta$-estradiol and progesterone were up-regulated with BAPT treatments. Moreover, the expression levels of Oct4, Bmp15, GDF9, FOXO3, Zp3, FOXL2, Inhibin alpha, SOD2, Catalase, GPX and BCl-2 in the developing follicles were significantly up-regulated after BAPT treatments $(P<0.05)$, while BAPT significantly inhibited the expression levels of BAX $(P<0.05)$. Following BAPT treatments, the ROS production levels of MII oocytes were decreased while the mitochondrial distributions were significantly enhanced. Furthermore, increased maturation rates, fertilization and embryonic developmental rates were found in these BAPT-treated groups $(P<$ 0.05).

These results demonstrated that BAPT significantly improved the development of preantral follicles in vitro by reducing ROS-dependent cellular damages and by enhancing mitochondrial distributions, thereby promoting the further applications of animal-derived BAPT in biomedical research.
\end{abstract}

Keywords: Preantral follicles, BAPT, ROS, Embryonic development

\section{Introduction}

Folliculogenesis, a major issue related with mammalian ovulation and fertility, occurs as a complex and dynamic process within female ovaries, which could be affected by multiple endocrine and intraovarian

\footnotetext{
*Correspondence: xlsu@sina.com; daiyf@imu.edu.cn

${ }^{\dagger}$ Gang Liu and Shubin Li contributed equally to this work.

${ }^{1}$ Key Laboratory of Medical Cell Biology, Clinical Medicine Research Center, Affiliated Hospital of Inner Mongolia Medical College, 1 Tongdao North Street, Hohhot 010050, Inner Mongolia, China

${ }^{2}$ College of Life Science, Inner Mongolia University, 235 West Univ. Road, Hohhot 010021, Inner Mongolia, China
}

paracrine factors including antioxidants, nutrients and vitamins [1]. The dysfunction of mammalian folliculogenesis, due to abnormalities in endocrine/paracrine signals, metabolic dysfunction, impaired oocyte developmental abilities or altered gene expression patterns, results in infertility-related diseases and reproductive disorders, such as ovarian cancer and polycystic ovary syndrome (PCOS) $[2,3]$.

Due to the progressing development of assisted reproductive biotechnologies, these culture and maturation models of follicles mimic the native environmental

(c) The Author(s). 2020 Open Access This article is licensed under a Creative Commons Attribution 4.0 International License, which permits use, sharing, adaptation, distribution and reproduction in any medium or format, as long as you give appropriate credit to the original author(s) and the source, provide a link to the Creative Commons licence, and indicate if changes were made. The images or other third party material in this article are included in the article's Creative Commons licence, unless indicated otherwise in a credit line to the material. If material is not included in the article's Creative Commons licence and your intended use is not permitted by statutory regulation or exceeds the permitted use, you will need to obtain permission directly from the copyright holder. To view a copy of this licence, visit http://creativecommons.org/licenses/by/4.0/ The Creative Commons Public Domain Dedication waiver (http://creativecommons.org/publicdomain/zero/1.0/) applies to the data made available in this article, unless otherwise stated in a credit line to the data. 
differences of the developing follicles in vitro to obtain the functional germ cells [4]. Moreover, the culture models of follicles in vitro allow the investigation of specific regulatory mechanisms related with in vivo folliculogenesis, as well as the search of diverse supplementary factors to sustain follicular development and the assessment of therapeutic agents against various reproductive disorders $[5,6]$.

However, the production and accumulation of reactive oxygen species (ROS) from cellular metabolic processes in the developing follicles affects the development and survival of follicles during long-term culture [7]. Indeed, when excessive ROS levels overwhelm the balance of the cellular antioxidant defense system, oxidative stress (OS) occurs $[8,9]$, resulting in direct or indirect ROSmediated damage to nucleic DNA, proteins and lipids, thereby altering their functions and resulting in the abnormal gene expression patterns, DNA double strand break (DSB) repair, chromosomal errors and follicular development [10-13].

Therefore, the addition of antioxidants to follicle cultures as ROS scavengers could prevent or decrease harmful damages, resulting in increased qualitiy of germ cells [14-16]. Although different antioxidants, such as rutin [17], $\beta$-mercaptoethanol [18] and $\alpha$ lipoic acid [19], have been routinely applied to the culture system of developing follicles, the effect of bioactive peptides (BAPT), natural antioxidants derived from various food or animals sources [20] on the development of mammalian preantral follicles has not yet been investigated.

BAPT were characterized as unique bioactive polypeptidic compounds with short sequences of approximately several or tens of amino acids [21,22]. With the rapid development of purification techniques including membrane filtration [23], gel filtration chromatography (GFC) [24], Ion-exchange chromatography (IEX) [25], ultra-high-performance liquid chromatography-tandem mass spectrometry (UHPLC-MS/MS) [26] and rapid resolution liquid chromatography-tandem mass spectrometry (RRLC-MS) [27], abundant BAPT species were purified and identified, leading to the various applications of BAPT, ranging from the development of biomaterials to therapeutic uses, in the past decade [28].

Furthermore, numerous studies have revealed that the biological activities of BAPT, including antioxidants, hormonal effects, immune system regulation, antithrombotic, anti-hypertensive, anti-bacterial, anti-viral and anti-cancer effects [29], depended on the original species, amino acid composition, and sequence of BAPT [30]. For instance, our previous studies demonstrated that animal-derived BAPT could suppressed the progression of colorectal cancer [31] and gastric cancer [32] in vivo without damages to normal cells. Nevertheless, little is known about the possible antioxidant effects of
BAPT on the mammalian reproductive system and follicle developmental proficiency.

Therefore, this study examined the effects of BAPT on the development of mouse preantral follicles and oocyte in vitro to assess the antioxidant effect of BPAT on the mammalian reproductive system.

\section{Results}

The effect of BAPT on the development of preantral follicles cultured in vitro.

To investigate the potential effect of BAPT treatment on the development of preantral follicles in vitro, the morphology and mean diameters of follicles cultured in vitro for 7, 14 and $21 \mathrm{~d}$ after BATP treatment were analyzed. As shown in Fig. 1 and Fig. 2, the results of follicular development in vitro showed that after cultured for $7 \mathrm{~d}$, the growth of isolated follicles was significantly promoted in all groups with the mean diameters of cultured follicles significant increased from $75.11 \pm$ $3.26 \mu \mathrm{m}$ in the control group, $86.22 \pm 3.38 \mu \mathrm{m}$ in the BAPT 20 group, $101.56 \pm 6.14 \mu \mathrm{m}$ in the BAPT 40 group to $112.78 \pm 6.20 \mu \mathrm{m}$ in the BAPT 60 group $(P<0.05)$, respectively.

On day 14, the diameter of follicles significantly increased from $133.66 \pm 6.85 \mu \mathrm{m}$ in the control group, $160.89 \pm 7.55 \mu \mathrm{m}$ in the BAPT 20 group, $187.22 \pm$ $7.36 \mu \mathrm{m}$ in the BAPT 40 group to $214.56 \pm 9.26 \mu \mathrm{m}$ in the BAPT 60 group $(P<0.05)$, respectively.

Furthermore, after $21 \mathrm{~d}$ in vitro culture, the mean diameter of follicles in the BAPT 40 group $(252.00 \pm$ $8.93 \mu \mathrm{m})$ and BAPT 60 group $(290.78 \pm 8.02 \mu \mathrm{m})$ were significantly higher than that of control group $(204.89 \pm$ $5.60 \mu \mathrm{m})$ and BAPT 20 group $(222.78 \pm 11.51 \mu \mathrm{m})(P<$ $0.05)$, which further indicated that BAPT treatment significantly enhanced the growth of isolated follicles in a dose-dependent manner.

\section{Hormonal assays}

As shown in Fig. 3, after $14 \mathrm{~d}$ of in vitro culture, the level of E2 in the control group $(20.70 \pm 1.10 \mathrm{ng} / \mathrm{mL})$ was significantly lower than those of BAPT treatment groups, respectively $(P<0.05)$. Indeed, the E2 levels were significantly increased from $23.45 \pm 1.54 \mathrm{ng} / \mathrm{mL}$ in the BAPT 20 group, $26.73 \pm 1.69 \mathrm{ng} / \mathrm{mL}$ in the BAPT 40 group to $32.41 \pm 1.50$ $\mathrm{ng} / \mathrm{mL}$ in the BAPT 60 group $(P<0.05)$.

Furthermore, after $14 \mathrm{~d}$ in vitro of culture, the level of P4 significantly increased from $17.98 \pm 1.03 \mathrm{ng} / \mathrm{mL}$ in the control group, $21.23 \pm 1.21 \mathrm{ng} / \mathrm{mL}$ in the BAPT 20 group, $25.51 \pm 1.20 \mathrm{ng} / \mathrm{mL}$ in the BAPT 40 group to $31.40 \pm 1.79 \mathrm{ng} / \mathrm{mL}$ in the BAPT 60 group $(P<0.05)$, respectively, which further showed that BAPT treatments significantly enhanced the functions of follicles in a dose-dependent manner. 


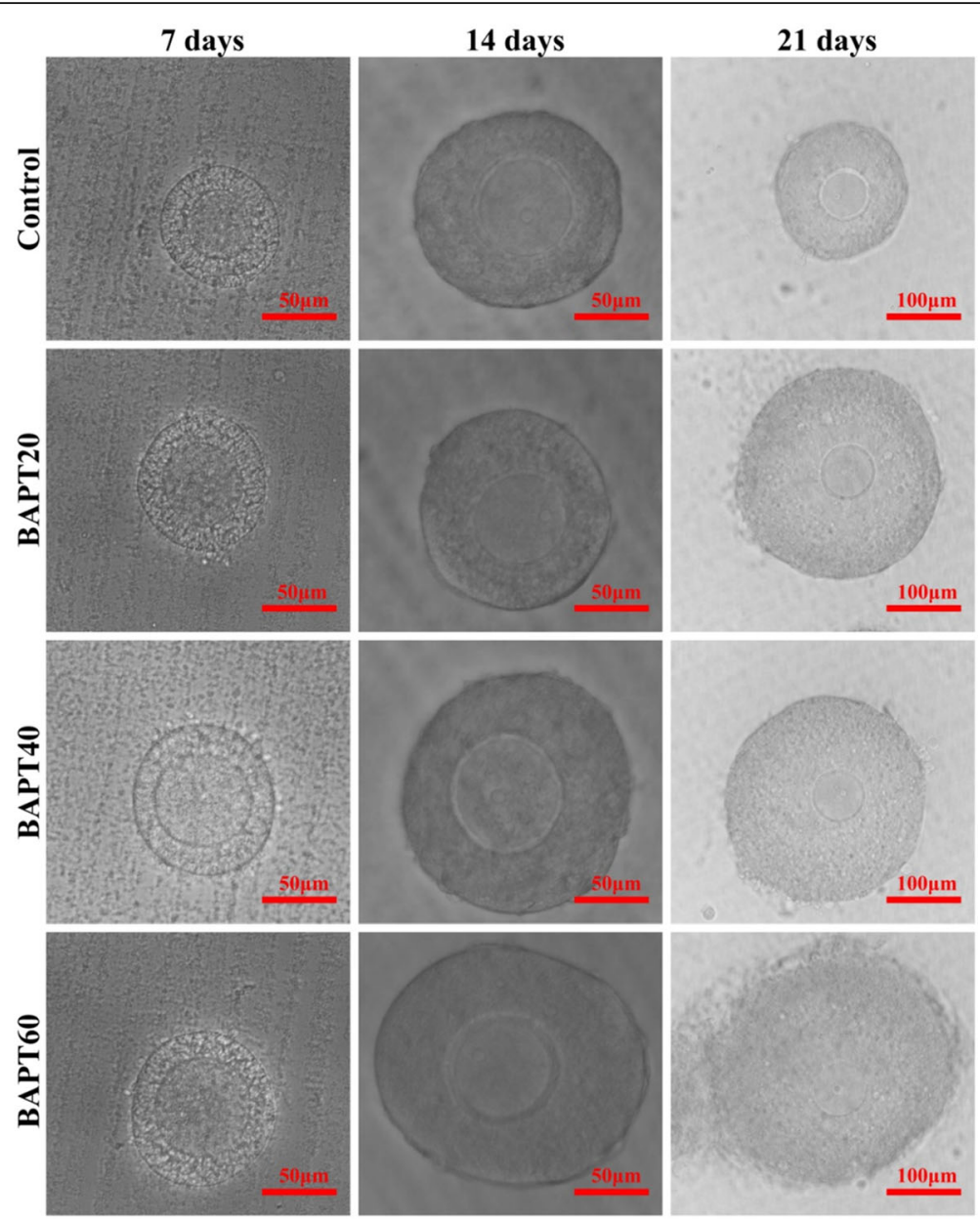

Fig. 1 The representative growth of follicles cultured in vitro after different periods

\section{Gene expression patterns of in vitro cultured follicle}

To assess the effect of BAPT treatment on the gene expression patterns of in vitro cultured follicle the expression levels of specific genes related to oogenesis (Oct4, Bmp15, GDF9, FOXO3 and Zp3), granulogenesis (FOXL2 and Inhibin alpha), ROS production (SOD2, Catalase and GPx) and apoptosis process ( $B A X$ and $B c l-2)$ in the developing follicles after $14 \mathrm{~d}$ of in vitro culture were analyzed by RT-PCR.

As shown in Fig. 4, BAPT treatments significantly promoted the relative expression levels of specific genes

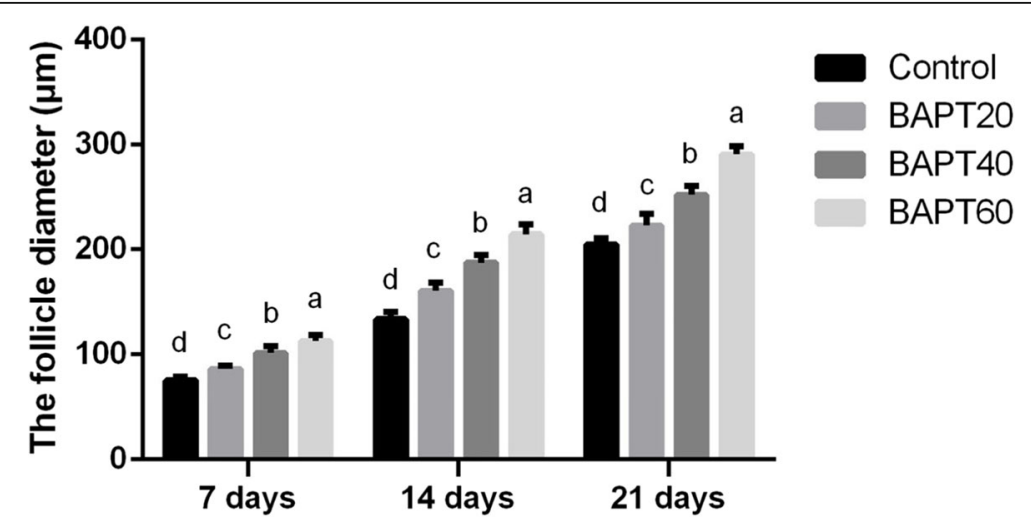

Fig. 2 The mean diameter of follicles cultured in vitro after different periods. Note: In each panel, labeling with the different letter in each column indicates significant differences between different groups $(P<0.05)$ 
A

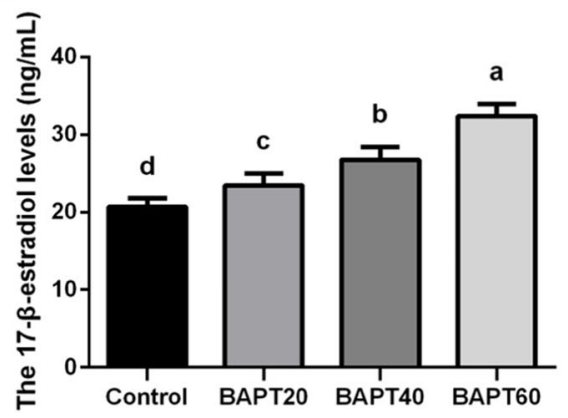

B

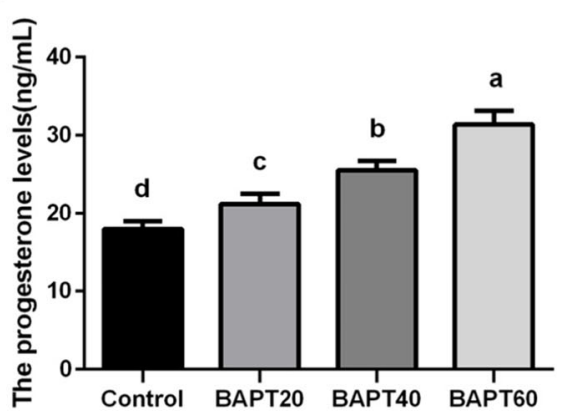

Fig. 3 The hormone levels of follicles cultured in vitro for $14 \mathrm{~d}$. A: E2 levels of follicles cultured in vitro after different periods; B: P4 levels of follicles cultured in vitro after different periods. Note: In each panel, labeling with the different letter in each column indicates significant differences between different groups $(P<0.05)$

related to oogenesis (Oct4, Bmp15, GDF9, FOXO3 and $Z p 3)$ and granulogenesis (FOXL2 and Inhibin alpha) in the developing follicles in a dose-dependent manner $(P<$ 0.05).

In addition, the relative expression levels of ROS production-related genes (SOD2, Catalase and GPX) in BAPT treatment groups were significantly up-regulated with the increasing doses of BAPT $(P<0.05)$, indicating that BAPT treatment significantly inhibited ROS production of in vitro cultured follicles.

On the other hand, the relative gene expression levels of $B A X$ in the BAPT 40 group and BAPT 60 group were significantly lower than that of control and BAPT 20 group $(P<0.05)$. However, after BAPT treatment, the relative gene expression levels of $\mathrm{Bcl}-2$ in all BAPT treated group were significantly higher than that in the control group $(P<0.05)$.

\section{The viability analyses of in vitro cultured follicles}

To investigate the effect of BAPT treatments on the viability of in vitro cultured follicles, the development of the follicles in different groups were analyzed by the measurement of viability rates, antrum formation rates and MII oocyte developmental rates calculated after 21 $\mathrm{d}$ of follicle culture. As shown in Fig. 5, the viabilities of follicles (Fig. 5A) and antrum formation rates (Fig. 5B) were significantly increased in the BAPT 40 group and BAPT 60 group in comparison with that of the control group and BAPT 20 group, respectively $(P<0.05)$.

After in vitro maturation, the developmental rate of MII oocytes (Fig. 5C) in the BAPT 60 group $(39.70 \pm 1.81 \%)$ was significantly higher than those of the control group $(22.20 \pm 1.66 \%)$, BAPT 20 group $(25.84 \pm 1.48 \%)$ and BAPT 40 group $(34.08 \pm 2.85 \%)$, respectively $(P<0.05)$.

\section{ROS production and mitochondrial distribution analyses of MIl oocytes}

To investigate the effect of BAPT treatments on the viability of oocytes, ROS production levels and mitochondrial distributions in MII oocytes of different groups were analyzed with the representative images of DCFH-

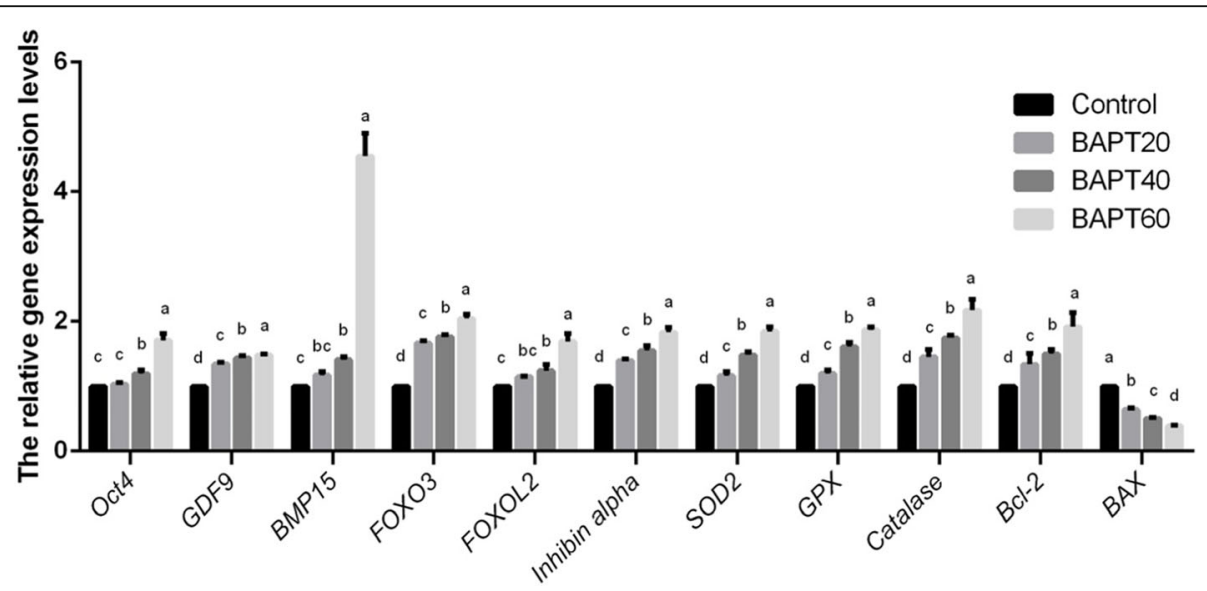

Fig. 4 The relative gene expression levels in follicles cultured in vitro for $14 \mathrm{~d}$. Note: Labeling with the different letter in each column indicates significant differences between different groups $(P<0.05)$ 

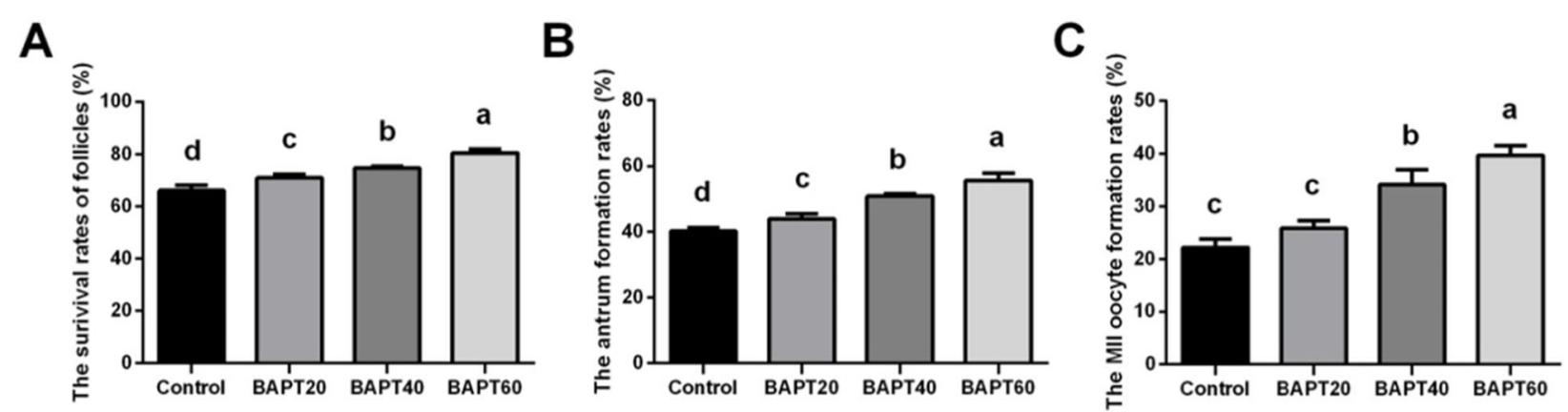

Fig. 5 The developmental rates of follicles cultured in vitro for $21 \mathrm{~d}$. A: The survival rates of follicles cultured in vitro for $21 \mathrm{~d}$; B: The antrum formation rates of follicles cultured in vitro for $21 \mathrm{~d}$; C: The MIl oocytes developmental rates of follicles cultured in vitro for $21 \mathrm{~d}$. Note: In each panel, labeling with the different letter in each column indicates significant differences between different groups $(P<0.05)$

DA fluorescence staining and mitochondrial distribution in MII oocytes shown in Fig. 6 and Fig. 7, respectively.

As shown in Fig. 6, the fluorescence intensities of DCFH-DA in MII oocytes were remarkably decreased in all BAPT treated groups compared with the control group $(P<0.05)$, indicating that BAPT treatment significantly inhibit ROS production process in MII oocytes during the long-term in vitro culture process of follicles. Moreover, the reduction of fluorescence intensity of DCFH-DA in MII oocytes was negatively related with the increasing concentration of BAPT $(P<0.05)$.

Furthermore, the fluorescence intensities of MitoTracker Red CMXRos in MII oocytes were remarkably increased in all BAPT treated groups compared with the control group $(P<0.05)$ (Fig. 7$)$, indicating that BAPT treatment significantly promoted the mitochondrial accumulation levels of MII oocytes during the long-term in vitro culture process of follicles.

\section{Fertilization rate and embryonic development analyses}

The functional analysis of MII oocytes to develop into embryos after fertilization is the gold standard for the examination of in vitro follicular cultures. To analysis the developmental potential of MII oocytes, the fertilization rates of MII oocytes and embryonic developmental rates in different BAPT groups were further measured. As shown in Fig. 8, the percentage of fertilization rates was remarkably increased in all BAPT treated groups $(P<0.05)$ with the highest percentage of fertilization rate found in the BAPT 60 group. Moreover, the embryonic developmental rates from 2-cell embryo, 8-cell embryo to blastocyst were significantly higher in all BAPT treated groups compared with the control group $(P<0.05)$.

\section{Discussion}

Here we provided the first evidence that BAPT promotes the growth of in vitro cultured preantral follicles and oocytes with remarkable up-regulation of oogenesis/ granulogenesis process and significant inhibition of cellular ROS production and apoptosis.

Under normal conditions, the generation and elimination of ROS are effectively controlled by the cellular antioxidant defense system [33,34], consisting of antioxidant enzymes (catalases in peroxisomes, glutathione peroxidases and superoxide dismutases in the cytosol), nonenzymatic factors (carnitine, coenzyme Q10, glutathione, pantothenic acid and vitamins) and micronutrients (copper, selenium and zinc) [35]. However, the unbalances between ROS production levels and the generation of free radicals under pathological conditions lead to oxidative damages to cellular proteins, membrane lipids and DNA, resulting in these health disorders including malignant cancer, coronary heart diseases and neuronal degeneration via dysregulted ATP generation, catabolic/anabolic processes and post-translational protein modifications [34, 36, 37]. Furthermore, the lipid oxidation mediated by excessive accumulation of free radicals interferes with these cellular amino acids, proteins, vitamins and cholesterol during the process of food preparation, transportation and storage, resulting into toxic compounds.

The increasing demand for effective antioxidants to delay the lipid oxidation in the pharmaceutical and health food industry promoted the development of antioxidants including butylated hydroxytoluene (BHT), butylated hydroxyanisole (BHA) and tertiary butylhydroquinone (TBHQ). However, these artificial antioxidants have been reported to be associated with liver damage and carcinogenesis. Therefore, the searching for a novel, natural and efficient antioxidant will promote the application of antioxidants in the functional and designer food industry.

During the promising investigation of natural antioxidants, BAPT have attracted increasing interest as prominent candidates. As a consequence, various products or ingredients with BAPT as fundamental constituents were commercially available and marketed globally, which 


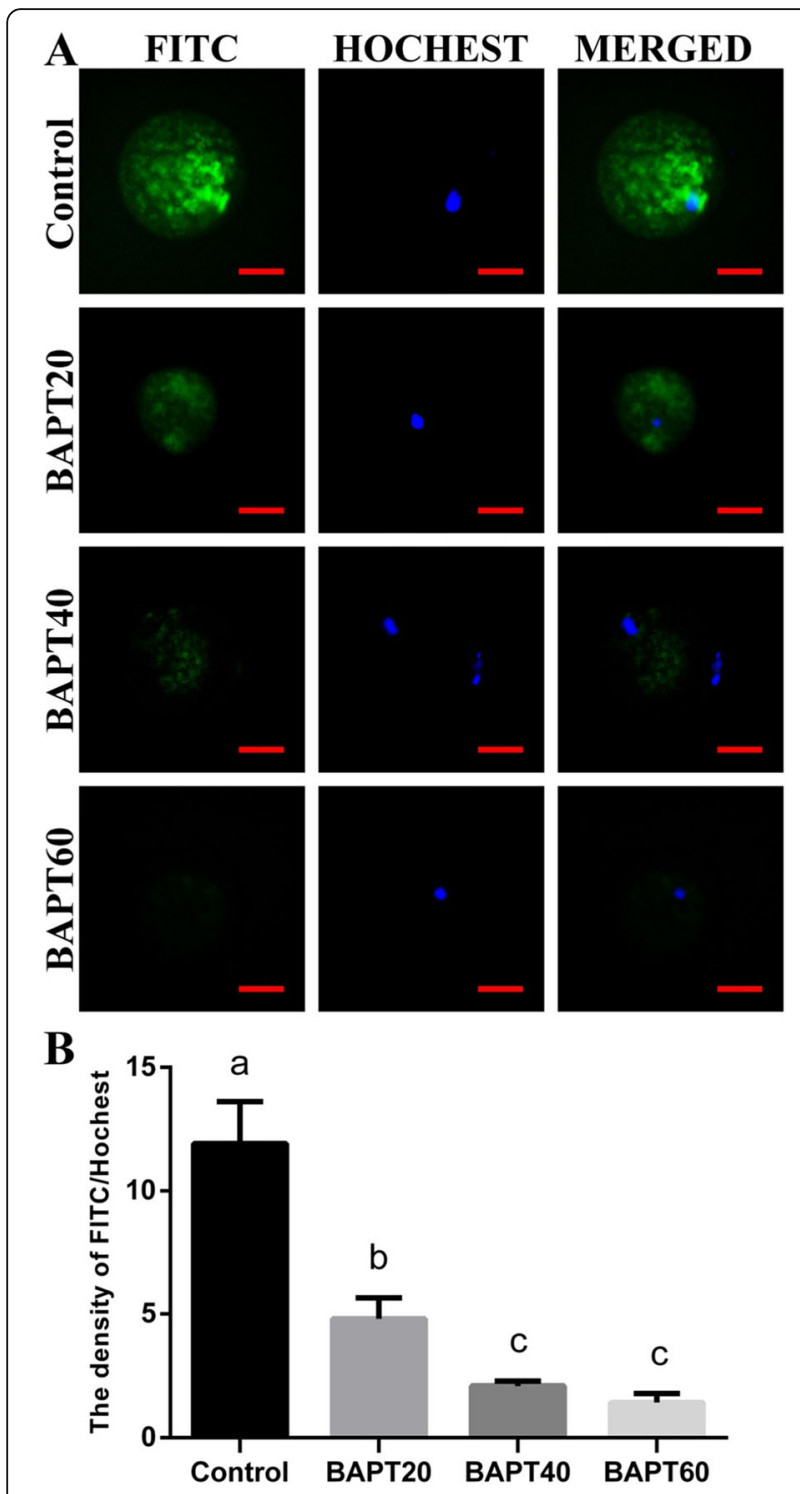

Fig. 6 The ROS production levels in MII oocytes from different groups. A: The representative ROS staining of MIl oocytes from different groups; Note: Scale bar $=30 \mu \mathrm{m}$. B: The relative density of FITC/ Hochest staining of MII oocytes from different groups. Note: Labeling with the different letter in each column indicates significant differences between different groups $(P<0.05)$

were based on the specific characteristics of BAPT including anticancer [38], antimicrobial [39], antihypertensive [40], antithrombotic [41], antioxidative [42], immunomodulatory [43] and cholesterol-lowering properties [44]. These products are enriched with BAPT either by modification of the usual manufacturing processes or by simply adding the BAPT during product preparation. Such BAPT-based functional food market is a fast-growing segment of the food industry and is still expanding thanks to the increasing awareness and purchasing power of consumers.

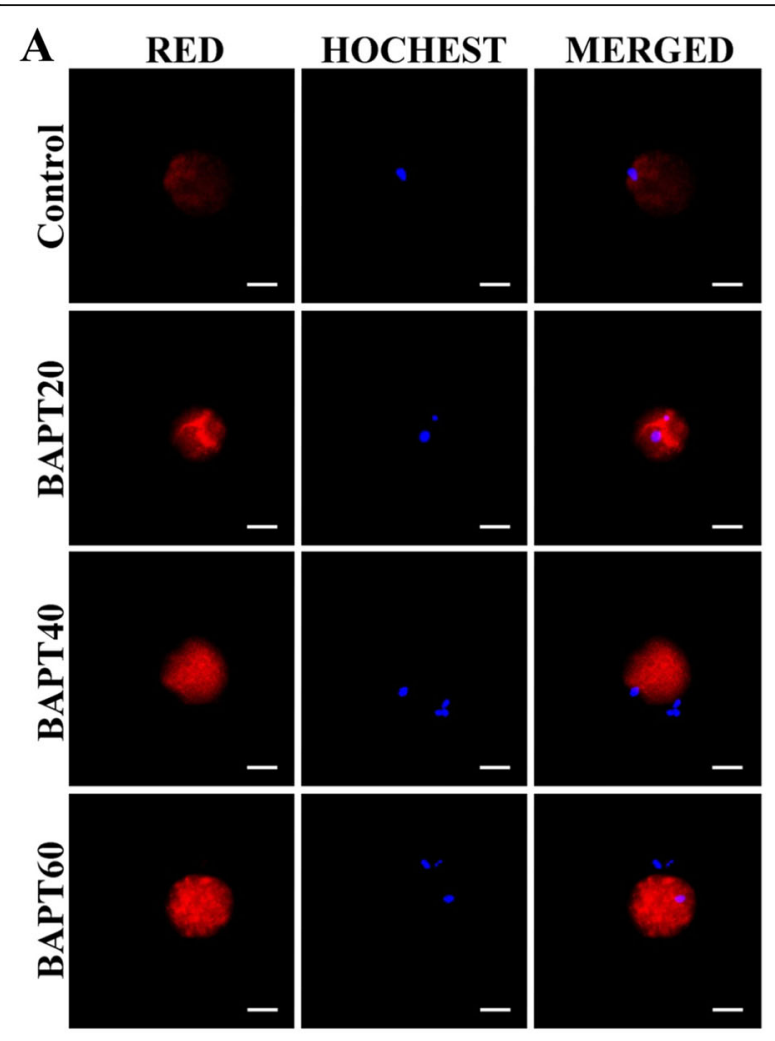

B

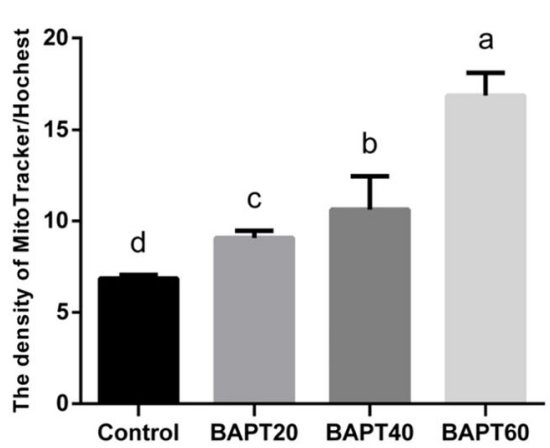

Fig. 7 The mitochondrial distributions in MIl oocytes from different groups. A: The representative mitochondrial staining results of MII oocytes from different groups; Note: Scale bar $=20 \mu \mathrm{m}$. B: The relative density of MitoTracker/Hochest staining of MII oocytes from different groups. Note: Labeling with the different letter in each column indicates significant differences between different groups $(P<0.05)$

In our study, BAPT was supplied as a natural antioxidant during in vitro culture of preantral follicles. We found that BAPT supplement significantly promoted the growth of in vitro cultured preantral follicles and oocyte qualities in a dose-dependent manner, as confirmed by the expression levels of reproduction-related genes in follicles and developmental rates of oocytes in vitro. Moreover, the growth promotion mechanisms were significantly associated with the reduction of ROS levels during follicle development. 

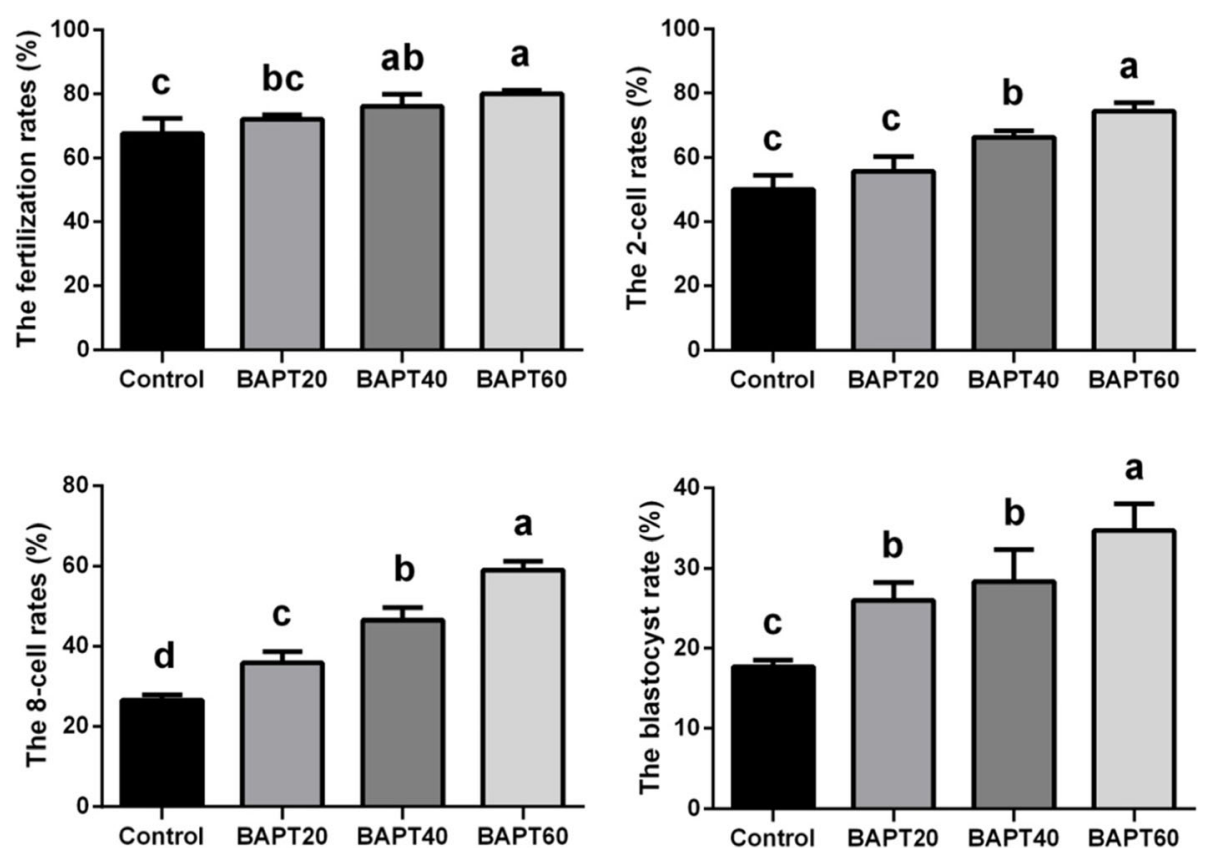

Fig. 8 The fertilization rates and embryonic developmental rates in different groups. Note: Labeling with the different letter in each column indicates significant differences between different groups $(P<0.05)$

Considering the growth of preantral follicles during the long-term culture process in vitro, the analyses of RT-PCR and hormone levels were conducted after 14 days of follicle culture [45, 46]. After BAPT treatment, the ROS production-related genes as SOD2, Catalase and GPx, which regulated the cellular antioxidant defense system, were significantly up-regulated with the increasing dose of BAPT, indicating that BAPT treatment significantly inhibited ROS production process of isolated follicles cultured in vitro.

During the past decades, numerous studies have confirmed that increased ROS levels were related with the disruptions of oocyte maturation and embryonic development through mitochondrial damages [47-49]. Therefore, to further confirm the effect of BAPT on the inhibition of ROS production in preantral follicles cultures, the mitochondrial distributions of MII oocytes were analyzed. We found that after BAPT treatment, higher APT synthesis was accompanied by the inhibition of ROS production, illustrating the function of BAPT in reducing ROS-dependent energetic impariments. As the ATP-generating organelles, mitochondria distributed around the nucleus play crucial roles in oocyte maturation, fertilization and early embryonic development. Increased mitochondrial distribution after BAPT treatment likely contributes to an enhanced meiotic progression of oocytes.

On the other hand, the increased expression levels of $B c l-2$ and decreased expression levels of Bax in follicles, which are considered as the biomarkers of inhibited cellular apoptosis process, were observed in BAPTtreated groups, demonstrating that BAPT treatment inhibit cellular apoptosis by regulating the expression of critical genes.

Therefore, the results of the present study have promising implications for biomedical research and warrant further in vivo studies. Future research should verify the role of BAPT in the prevention and treatment of other infertility disorders, such as PCOS, ovarian aging and clinical infertilities. In addition, the effect of BAPT on follicle's gene expression and hormone expression levels after long-term culture process than $21 \mathrm{~d}$ needs further explorations.

\section{Conclusions}

In conclusion, bioactive peptides (BAPT) from animal sources significantly improved follicular development throughout long-term in vitro culture of mouse preantral follicles. During the culture process, BAPT treatment reduced cellular damage caused by ROS production and promoted the mitochondrion levels in follicles with significantly increased maturation rates, fertilization and embryonic developmental rates in vitro.

\section{Methods}

\section{Chemicals}

Unless otherwise stated, all chemicals, media and supplements used in this study were purchased from Sigma Aldrich (Shanghai, China). The BAPT with the special characteristic of ubiquitin enzyme and molecular weight 
as $8 \mathrm{Kd}$ were derived from spleen of small-tailed-Han sheep and identified by Professor Su's research group at Inner Mongolia Medical University (Hohhot, China) with the RRLC-MS method [22, 50-52].

Briefly, five small-tailed-Han sheep (2-year-old females; Inner Mongolia Dumei Animal Husbandry Biotechnology Co., Ltd) were housed at $22-24{ }^{\circ} \mathrm{C}$ with humidity at $45-50 \%$ and a natural light/dark cycle. Standard mineral blocks and water were offered to animals ad libitum. After animal sacrifice, the spleen was obtained and rapidly pulverized.

After pulverization, the spleen mixtures were homogenized with normal saline at a ratio of $0.125-0.5 \mathrm{~g} / \mathrm{mL}$, followed by frozen and thaw under $-80^{\circ} \mathrm{C}$ for three times. After filtration, the supernatants were centrifuged under $4{ }^{\circ} \mathrm{C}$ at $14000 \times \mathrm{g}$ for $20 \mathrm{~min}$. After centrifugation, the supernatants were collected and BAPT was isolated with the RRLC-MS method.

Animals, follicle isolation and experimental design.

A total of 50 6-week-old females mice of the Institute of Cancer Research (ICR) strain (Beijing Vital River Laboratory Animal Technology Co., Ltd., 201) were used for this study. The ICR mice were housed in a controlled environment at $21{ }^{\circ} \mathrm{C}, 53$ to $63 \%$ relative humidity with 12-h light/dark cycles. Standard feed and water were provided ad libitum.

The ICR mice were sacrificed by cervical dislocation; subsequently, bilateral ovaries were dissected and placed immediately into fresh medium as $\alpha$ lpah-minimal essential medium ( $\alpha$-MEM) (12,571,063, Gibco, Shanghai, China) supplemented with $10 \%$ fetal bovine serum (FBS) (10099141C, Gibco, Shanghai, China). The follicles were isolated mechanically under a stereomicroscope $(745 \mathrm{~T}$, Nikon, Tokyo, Japan) with a 26-gauge needle, then intact preantral follicles displaying two or three layers of granular cells (GC) with normal and centrally located oocytes were collected [53]. These collected preantral follicles were rinsed twice and transferred into a culture dish with fresh basic culture medium.

The preantral follicles $(n=1198)$ were randomly divided into the following four groups based on the dose of supplemented BAPT to the basic culture medium. The control group $(n=289)$ was not treated with any BAPT, while the BAPT $20(n=310)$, BAPT $40(n=297)$ and BAPT 60 groups $(n=302)$ were supplemented with 20, 40 and $60 \mu \mathrm{g} / \mathrm{mL}$ BAPT, respectively. The optimization of BAPT concentration applied in this study was performed in our former study [50].

\section{In vitro culture of preantral follicles}

The isolated preantral follicles were cultured on an insert (PICM0RG50, Millipore, USA) in 6-well culture dishes (Corining, China) in $1200 \mu \mathrm{L}$ culture medium as $\alpha$-MEM medium supplemented with $2 \%$ FBS, $1 \times$
Glutamax (35,050,061, Gibco, Shanghai, China), 0.055 $\mathrm{mM}$ 2-mercaptoethanol (M6250, Sigma, Shanghai, China), $100 \mathrm{IU} / \mathrm{mL}$ follicle stimulating hormone (FSH) (Sansheng, Ningbo, China) and $100 \mathrm{IU} / \mathrm{mL}$ penicillin/ streptomycin (P/S, 15140122, Gibco, Shanghai, China) for $21 \mathrm{~d}$ in a $\mathrm{CO}_{2}$ incubator $\left(37^{\circ} \mathrm{C}\right.$ and $\left.5 \% \mathrm{CO}_{2}\right)$. Half of the culture medium was replaced by fresh culture medium every other day.

After in vitro follicle culture for different periods $(7 \mathrm{~d}$, $14 \mathrm{~d}$ and $21 \mathrm{~d}$ ), the morphologies of the cultured follicles were examined and recorded with an inverted fluorescence microscope (Ti2, Nikon, Tokyo, Japan). Images of the cultured follicles were randomly taken for each group at a $10 \times$ magnification. The mean diameters of the developing follicle were analyzed through Image J software ( $n=10$ for each group).

\section{Hormonal assays}

After in vitro follicle culture for $14 \mathrm{~d}$, half of each follicle culture medium was collected to investigate the sex hormone levels of $17 \beta$-estradiol (E2) and progesterone (P4) with a commercial estradiol ELISA kit (PE223, Beyotime, Shanghai, China) and a commercial progesterone ELISA kit (PP773, Beyotime, Shanghai, China), respectively.

\section{Reverse transcription PCR and real-time PCR analyses}

After in vitro follicle culture for $14 \mathrm{~d}$, total RNA from cultured follicles $(n=30)$ of different groups was extracted with Trizol reagent (79,306, Gibco, Shanghai, China), respectively. The synthesis of cDNA was conducted according to the manufacturer's instructions (Prime Script $^{\mathrm{m}}$ RT reagent kit with gDNA Eraser, RR047A, Takara, Dalian, China). For PCR amplification, specific primers were designed on the NCBI website and commercially synthesized by Invitrogen (Shanghai, China). Real-time PCR was performed within a Thermo Scientific PikoReal system with a commercial kit (RR820A, Premix Taq ${ }^{\mathrm{m}}$, Takara, Dalian, China).

PCR reactions started with an initial melting step for 5 min at $95^{\circ} \mathrm{C}$, followed by 35 cycles of melting at $95^{\circ} \mathrm{C}$ for $30 \mathrm{~s}$, annealing at $58^{\circ} \mathrm{C}$ for $30 \mathrm{~s}$ and elongation at $72{ }^{\circ} \mathrm{C}$ for $30 \mathrm{~s}$. The ubiquitously expressed $\beta$-actin gene was used as an internal control. The qualities of PCR reactions were confirmed by the melting curve analyses with all experiments performed in triplicate. The relative mRNA expression was calculated using the $2^{-\Delta \Delta C t}$ method [54].

The primers for Reverse Transcription PCR and Realtime PCR analyses were as follows:

Oct4.

Forward primer-GATGCTGTGAGCCAAGGCAAG; Reverse primer-GGCTCCTGATCAACAGCATCAC; Bmp15.

Forward primer-TCCTTGCTGACGACCCTACAT; 
Reverse primer-TACCTCAGGGGATAGCCTTGG; GDF9.

Forward primer-TCTTAGTAGCCTTAGCTCTCAGG;

Reverse primer-TGTCAGTCCCATCTACAGGCA;

FOXO3.

Forward primer-CTGGGGGAACCTGTCCTATG;

Reverse primer-TCATTCTGAACGCGCATGAAG;

FOXL2.

Forward primer-ACAACACCGGAGAAACCAGAC;

Reverse primer-CGTAGAACGGGAACTTGGCTA;

Inhibin alpha.

Forward primer-GCACAGGACCTCTGAACCAG;

Reverse primer-GGGATGGCCGGAATACATAAG;

SOD2.

Forward primer-ATGGTGGGGGACATATT;

Reverse primer-GAACCTTGGACTCCCACAGA;

Catalase.

Forward primer-CCTCGTTCAGGATGTGGTTT;

Reverse primer-TCTGGTGATATCGTGGGTGA;

$G P x$

Forward primer-GTCCACCGYGTATGCCTTCT;

Reverse primer-TCTGCAGATCGTTCATCTCG;

$B A X$

Forward primer-TGAAGACAGGGGCCTTTTTG;

Reverse primer-AATTCGCCGGAGACACTCG;

Bcl-2.

Forward primer-ATGCCTTTGTGGAACTATATGGC;

Reverse primer-GGTATGCACCCAGAGTGATGC;

$\beta$-actin.

Forward primer-GGCTGTATTCCCCTCCATCGT;

Reverse primer-TGGTGCCAGATCTTCTCCATGTC.

\section{In vitro maturation}

After in vitro follicle culture for $21 \mathrm{~d}$, the developing follicles of each group were mechanically dissected by with a pulled bore glass capillary with the released cumulus cell-oocyte complexes (COC) collected for in vitro maturation (IVM).

The collected COCs were then cultured in a 4-well culture dish (Nunc, China) in $500 \mu \mathrm{L} \alpha$-MEM medium supplemented with 5\% FBS, $0.1 \mathrm{IU} / \mathrm{mL}$ FSH, $1.2 \mathrm{IU} /$ $\mathrm{mL}$ human chorionic gonadotropin (HCG, Sansheng, Ningbo, China), $4 \mathrm{ng} / \mathrm{mL}$ epidermal growth factor (EGF, 315-09, Proteintech, Shanghai, China), $1.5 \mathrm{mM}$ ascorbic acid (A4403, Sigma, Shanghai, China) and $100 \mathrm{IU} / \mathrm{mL}$ P/S covered in mineral oil (M8410, Sigma, Shanghai, China) in a $\mathrm{CO}_{2}$ incubator $\left(37^{\circ} \mathrm{C}\right.$ and $5 \%$ $\mathrm{CO}_{2}$ ).

After $17 \mathrm{~h}$ of culture, oocytes from the expanded COCs were further examined microscopically for the maturation with first polar bodies. The maturation rates of each group were further analyzed with the maturation rates calculated as the percentage of maturing oocytes for each developing follicles [54].

\section{Analyses of ROS levels in MII oocytes}

MII oocytes from different groups were collected and incubated in CZB medium containing $10 \mu \mathrm{M}$ dichlorofluorescein diacetate (DCFH-DA, S0033, Beyotime, Shanghai, China) for $30 \mathrm{~min}$ at $37^{\circ} \mathrm{C}$ according to the manufacturer's protocols. After incubation, MII oocytes were re-stained with $5 \mu \mathrm{g} / \mathrm{mL}$ Hoechst 33432 solution (C0030, Solarbio, Beijing, China) for $10 \mathrm{~min}$ at $37^{\circ} \mathrm{C}$. The fluorescence staining density of oocyte were analyzed by confocal microscopy (A1R, Nikon, Tokyo, Japan).

Mitochndrial distribution analyses of MII oocytes.

MII oocytes from different groups were collected, rinsed three times with Dulbecco's phosphate buffered solutions (DPBS, 14190136, Gibco, Shanghai, China) and incubated in $4 \%$ paraformaldehyde solution (P1110, Solarbio, Beijing, China) at room temperature for 30 min, subsequently stained with $200 \mathrm{nM}$ Mito-Tracker Red CMXRos (C1049, Beyotime, Shanghai, China) for $30 \mathrm{~min}$ at $37^{\circ} \mathrm{C}$ according to the manufacturer's protocols. After incubation, MII oocytes were further restained with $5 \mu \mathrm{g} / \mathrm{mL}$ Hoechst 33432 solution at room temperature for $10 \mathrm{~min}$ and mounted on microscope slides for subsequent confocal microscopy.

\section{IVF and embryo culture}

To perform in vitro fertilization (IVF), the cauda epididymis of male ICR mice (ten weeks old) was dissected and placed immediately into the human tubal fluid (HTF) medium supplemented with $4 \mathrm{mg} / \mathrm{mL}$ BSA for $1 \mathrm{~h}$ in a $\mathrm{CO}_{2}$ incubator $\left(37^{\circ} \mathrm{C}\right.$ and $\left.5 \% \mathrm{CO}_{2}\right)$ for sperm capacitation. After sperm capacitation, the collected MII oocytes from different groups were transferred to HTF medium containing $1-2.5 \times 10^{6}$ capacitated spermatozoa and incubated for $6 \mathrm{~h}$ in a $\mathrm{CO}_{2}$ incubator $\left(37^{\circ} \mathrm{C}\right.$ and $\left.5 \% \mathrm{CO}_{2}\right)$. After insemination, the embryos were transferred to the Embryo ${ }^{\mathrm{Max}}$ KSOM medium (MR-020P-D, Sigma, Shanghai, China), which was supplemented with $1 \mathrm{mg} / \mathrm{mL}$ of BSA and covered by mineral oil in a $35 \mathrm{~mm}$ sterile plate (Corning). The embryos were cultured in vitro for $3.5 \mathrm{~d}$. During this period, the embryonic developmental stages (2-cell embryo, 8-cell embryo and blastocyst stage) of different groups were microscopically observed and recorded with the embryonic development rates calculated as percentage of embryo/ fertilized oocytes.

\section{Statistical analysis}

The analyses of results were carried out with the Statistical Package for the Social Sciences (SPSS, IBM, version19.0). All results are presented as mean \pm SD and assessed with one-way ANOVA LSD tests. $P<0.05$ was considered as significant. 


\section{Abbreviations}

BAPT: Bioactive peptides; BHA: Butylated hydroxyanisole; BHT: Butylated hydroxytoluene; COC: Cumulus cell-oocyte complex; DSB: DNA double strand break; FBS: Fetal bovine serum; FSH: Follicle stimulating hormone; GC: Granular cells; GFC: Gel filtration chromatography; HTF: Human tubal fluid; IEX: Ion-exchange chromatography; IVF: In vitro fertilization; IVM: In vitro maturation; OS: Oxidative stress; PCOS: Polycystic ovary syndrome; ROS: Reactive oxygen species; RRLC-MS: Rapid resolution liquid chromatography-tandem mass spectrometry; SPSS: Statistical Package for the Social Sciences; TBHQ: Tertiary butylhydroquinone; UHPLC-MS/MS: Ultra-highperformance liquid chromatography-tandem mass spectrometry

\section{Authors' contributions}

Data curation, Gang Liu, Shubin Li, and Jinyu Ren; Formal analysis, Gang Liu, Shubin Li, Chunyu Wang and Jinyu Ren; Funding acquisition, Xiulan Su and Yanfeng Dai; Investigation, Gang Liu, Shubin Li, Chunyu Wang, Yaxuan Zhang and Jinyu Ren; Methodology, Gang Liu, Shubin Li; Project administration, Yanfeng Dai; Resources, Xiulan Su and Yanfeng Dai; Supervision, Gang Liu and Yanfeng Dai; Writing-original draft, Gang Liu and Yanfeng Dai; Writing-review \& editing, Gang Liu. The author(s) read and approved the final manuscript.

\section{Funding}

This work was supported by the National Natural Science Foundation of China (81960560 to Xiulan Su) and the Inner Mongolia Science and Technology Research Project (2020BS08014 to Gang Liu, 2017ZD04 to Yanfeng Dai and CGZH2018149 to Xiulan Su).

\section{Availability of data and materials}

We declared that materials described in the manuscript, including all relevant raw data, will be freely available to any scientist wishing to use them for non-commercial purposes, without breaching participant confidentiality.

\section{Ethics approval and consent to participate}

The permission of this study was granted by the Animal Care Committee of Inner Mongolia University (IACUC Issue No.20190601-Gang Liu).

\section{Consent for publication}

This manuscript was an original research that has not been published previously, and not under consideration for publication elsewhere, in whole or in part.

\section{Competing interests}

The authors report no conflicts of interest in this work.

Received: 30 May 2020 Accepted: 28 July 2020

Published online: 15 September 2020

\section{References}

1. Dumesic DA, Padmanabhan V, Abbott DH. Polycystic ovary syndrome and oocyte developmental competence. Obstetrical \& gynecological survey. 2008;63(1):39-48.

2. Dewailly D, Robin G, Peigne M, Decanter C, Pigny P, Catteau-Jonard S. Interactions between androgens, FSH, anti-Mullerian hormone and estradiol during folliculogenesis in the human normal and polycystic ovary. Hum Reprod Update. 2016;22(6):709-24.

3. Qiao J, Feng HL. Extra- and intra-ovarian factors in polycystic ovary syndrome: impact on oocyte maturation and embryo developmental competence. Hum Reprod Update. 2011;17(1):17-33.

4. Aguiar FL, Lunardi FO, Lima LF, Rocha RM, Bruno JB, Magalhaes-Padilha DM, et al. Insulin improves in vitro survival of equine preantral follicles enclosed in ovarian tissue and reduces reactive oxygen species production after culture. Theriogenology. 2016;85(6):1063-9.

5. He X, Toth TL. In vitro culture of ovarian follicles from Peromyscus. Semin Cell Dev Biol. 2017;61:140-9.

6. Green LJ, Shikanov A. In vitro culture methods of preantral follicles. Theriogenology. 2016;86(1):229-38.

7. Carvalho AA, Faustino LR, Silva CM, Castro SV, Lobo CH, Santos FW, et al. Catalase addition to vitrification solutions maintains goat ovarian preantral follicles stability. Res Vet Sci. 2014;97(1):140-7.
8. Agarwal A, Gupta S, Sharma R. Oxidative stress and its implications in female infertility - a clinician's perspective. Reprod BioMed Online. 2005;11(5):641-50.

9. Lushchak VI. Free radicals, reactive oxygen species, oxidative stress and its classification. Chem Biol Interact. 2014;224:164-75.

10. Winship AL. Stringer JM. Hutt KJ. The importance of DNA repair for maintaining oocyte quality in response to anti-cancer treatments, environmental toxins and maternal ageing. Human reproduction update: Liew SH; 2018.

11. Leal ESS, Vieira LA, Sa NAR, Silva GM, Lunardi FO, Ferreira ACA, et al. In vitro growth and development of isolated secondary follicles from vitrified caprine ovarian cortex. Reprod Fertil Dev. 2018;30(2):359-70.

12. Kala M, Shaikh MV, Nivsarkar M. Equilibrium between anti-oxidants and reactive oxygen species: a requisite for oocyte development and maturation. Reproductive medicine and biology. 2017;16(1):28-35.

13. Tarin JJ, Vendrell FJ, Ten J, Blanes R, van Blerkom J, Cano A. The oxidizing agent tertiary butyl hydroperoxide induces disturbances in spindle organization, c-meiosis, and aneuploidy in mouse oocytes. Mol Hum Reprod. 1996;2(12):895-901.

14. Agarwal A, Majzoub A. Role of antioxidants in assisted reproductive techniques. The world journal of men's health. 2017;35(2):77-93.

15. Al-Gubory KH, Fowler PA, Garrel C. The roles of cellular reactive oxygen species, oxidative stress and antioxidants in pregnancy outcomes. Int J Biochem Cell Biol. 2010;42(10):1634-50.

16. Devine PJ, Perreault SD, Luderer U. Roles of reactive oxygen species and antioxidants in ovarian toxicity. Biol Reprod. 2012;86(2):27.

17. Lins T, Cavalcante AYP, Santos JMS, Menezes VG, Barros VRP, Barberino RS, et al. Rutin can replace the use of three other antioxidants in the culture medium, maintaining the viability of sheep isolated secondary follicles. Theriogenology. 2017;89:263-70.

18. Gupta PS, Nandi S. Viability and growth of buffalo preantral follicles and their corresponding oocytes in vitro: effect of growth factors and beta mercaptoethanol. Reproduction in domestic animals =. Zuchthygiene. 2010;45(1):147-54.

19. Talebi A, Zavareh S, Kashani MH, Lashgarbluki T, Karimi I. The effect of alpha lipoic acid on the developmental competence of mouse isolated preantral follicles. J Assist Reprod Genet. 2012;29(2):175-83.

20. Hamley IW. Small bioactive peptides for biomaterials design and therapeutics. Chem Rev. 2017;117(24):14015-41.

21. Ryder K, Bekhit Ael D, McConnell M, Carne A. Towards generation of bioactive peptides from meat industry waste proteins: generation of peptides using commercial microbial proteases. Food Chem. 2016;208:42-50.

22. Yu L, Yang L, An W, Su X. Anticancer bioactive peptide-3 inhibits human gastric cancer growth by suppressing gastric cancer stem cells. J Cell Biochem. 2014;115(4):697-711.

23. Vandanjon L, Johannsson R, Derouiniot M, Bourseau P, Jaouen P. Concentration and purification of blue whiting peptide hydrolysates by membrane processes. J Food Eng. 2007:83(4):581-9.

24. Zhang $Y$, Duan $X$, Zhuang Y. Purification and characterization of novel antioxidant peptides from enzymatic hydrolysates of tilapia (Oreochromis niloticus) skin gelatin. Peptides. 2012;38(1):13-21.

25. Levison PR. Large-scale ion-exchange column chromatography of proteins. Comparison of different formats. J Chromatogr B Anal Technol Biomed Life Sci. 2003;790(1-2):17-33.

26. Rizzetti TM, de Souza MP, Prestes OD, Adaime MB, Zanella R. A simple and fast method for the determination of 20 veterinary drug residues in bovine kidney and liver by ultra-high-performance liquid chromatography tandem mass spectrometry. Food Anal Method. 2017;10(4):854-64.

27. Li J, Mao C, Li L, Ji D, Yin F, Lang Y, et al. Pharmacokinetics and liver distribution study of unbound curdione and curcumol in rats by microdialysis coupled with rapid resolution liquid chromatography (RRLC) and tandem mass spectrometry. J Pharm Biomed Anal. 2014;95:146-50.

28. Daliri EB, Lee BH, Oh DH. Current trends and perspectives of bioactive peptides. Crit Rev Food Sci Nutr. 2018;58(13):2273-84.

29. Lafarga T, Hayes M. Bioactive peptides from meat muscle and by-products: generation, functionality and application as functional ingredients. Meat Sci. 2014;98(2):227-39

30. Wang X, Yu H, Xing R, Li P. Characterization, preparation, and purification of marine bioactive peptides. Biomed Res Int. 2017;2017:9746720.

31. Su LY, Shi YX, Yan MR, Xi Y, Su XL. Anticancer bioactive peptides suppress human colorectal tumor cell growth and induce apoptosis via modulating the PARP-p53-Mcl-1 signaling pathway. Acta Pharmacol Sin. 2015;36(12):1514-9.

32. Xing Z, Yu L, Li X, Su X. Anticancer bioactive peptide-3 inhibits human gastric cancer growth by targeting miR-338-5p. Cell \& bioscience. 2016;6:53. 
33. Tao J, Zhao YQ, Chi CF, Wang B. Bioactive Peptides from Cartilage Protein Hydrolysate of Spotless Smoothhound and Their Antioxidant Activity In Vitro. Marine drugs. 2018;16(4).

34. Gogineni V, Hamann MT. Marine natural product peptides with therapeutic potential: chemistry, biosynthesis, and pharmacology. Biochimica et biophysica acta General subjects. 2018;1862(1):81-196.

35. Walczak-Jedrzejowska R, Wolski JK, Slowikowska-Hilczer J. The role of oxidative stress and antioxidants in male fertility. Central European journal of urology. 2013;66(1):60-7.

36. Harnedy PA, O'Keeffe MB, FitzGerald R. Fractionation and identification of antioxidant peptides from an enzymatically hydrolysed Palmaria palmata protein isolate. Food research international (Ottawa, Ont). 2017;100(Pt 1):416-22.

37. Rahal A, Kumar A, Singh V, Yadav B, Tiwari R, Chakraborty S, et al. Oxidative stress, prooxidants, and antioxidants: the interplay. Biomed Res Int. 2014;2014:761264.

38. Qin Y, Zhou J, Zhang W, Yang X, Wang J, Wei C, et al. Construction of An anticancer fusion peptide (ACFP) derived from Milk proteins and An assay of anti-ovarian Cancer cells in vitro. Anti Cancer Agents Med Chem. 2016;17.

39. Porta A, Petrone A, Morello S, Granata I, Rizzo F, Memoli D, et al. Design and expression of peptides with antimicrobial activity against Salmonella Typhimurium: a procedure to identify peptides with AMP activity. Cell Microbiol. 2016;19.

40. Dabarera M, Ahiththan L, Perera PPR. Antihypertensive peptides from curd. AYU (An International Quarterly Journal of Research in Ayurveda). 2015;36:214.

41. Shiratsuchi E, Ura M, Nakaba M, Maeda I, Okamoto K. Elastin peptides prepared from piscine and mammalian elastic tissues inhibit collageninduced platelet aggregation and stimulate migration and proliferation of human skin fibroblasts. Journal of peptide science : an official publication of the European Peptide Society. 2010;16:652-8.

42. Kongcharoen A, Poolex W, Wichai T, Boonsombat R. Production of an antioxidative peptide from hairy basil seed waste by a recombinant Escherichia coli. Biotechnol Lett. 2016;38.

43. Blaurock N, Schmerler D, Hünniger K, Kurzai O, Ludewig K, Baier M, et al. Cterminal Alpha-1 antitrypsin peptide: a new Sepsis biomarker with Immunomodulatory function. Mediat Inflamm. 2016;2016:1-13.

44. Iwaniak A, Darewicz M, Minkiewicz P, Protasiewicz M, Borawska-Dziadkiewicz J. Biologically active peptides derived from food proteins as the food components with cardioprotective properties. Polski merkuriusz lekarski. 2014:36:403-6.

45. Tanaka Y, Matsuzaki T, Tanaka N, Iwasa T, Kuwahara A, Irahara M. Activin effects on follicular growth in in vitro preantral follicle culture. J Med Invest. 2019;66(1.2):165-71.

46. Kim IW, Gong SP, Yoo CR, Choi JH, Kim DY, Lim JM. Derivation of developmentally competent oocytes by the culture of preantral follicles retrieved from adult ovaries: maturation, blastocyst formation, and embryonic stem cell transformation. Fertil Steril. 2009:92(5):1716-24.

47. van Blerkom J, Davis P, Lee J. Fertilization and early embryolgoy: ATP content of human oocytes and developmental potential and outcome after in-vitro fertilization and embryo transfer. Hum Reprod. 1995;10:415-24.

48. Zhu C-C, Zhang Y, Duan X, Han J, Shao S. Toxic effects of HT-2 toxin on mouse oocytes and its possible mechanisms. Arch Toxicol. 2015;90.

49. Prather RS, Brown AN, Spate L, Redel B, Whitworth K. Whyte J. Control of Pig Reproduction IX: Transcriptional profiling of oocyte maturation and embryonic development elucudates metabolism and control of development; 2013.

50. Li X, Xia L, Ouyang X, Suyila Q, Su L, Su X. Bioactive peptides sensitize cells to anticancer effects of Oxaliplatin in human colorectal Cancer Xenografts in nude mice. Protein and peptide letters. 2019;26(7):512-22.

51. Su L, Xu G, Shen J, Tuo Y, Zhang X, Jia S, et al. Anticancer bioactive peptide suppresses human gastric cancer growth through modulation of apoptosis and the cell cycle. Oncol Rep. 2010;23(1):3-9.

52. Chen C, Su X, Hu Z. Immune promotive effect of bioactive peptides may be mediated by regulating the expression of SOCS1/miR-155. Exp Ther Med. 2019;18(3):1850-62.

53. Zhang C, Wang X, Wang Z, Niu W, Zhu B, Xia G. Effect of different culture systems and 3, 5, 3'-triiodothyronine/follicle-stimulating hormone on preantral follicle development in mice. PLoS One. 2013;8(4):e61947.

54. Liu G, Li S, Yuan H, Hao M. Wurihan, Yun Z, et al. effect of sodium alginate on mouse ovary vitrification. Theriogenology. 2018;113:78-84.

\section{Publisher's Note}

Springer Nature remains neutral with regard to jurisdictional claims in published maps and institutional affiliations.

\section{Ready to submit your research? Choose BMC and benefit from:}

- fast, convenient online submission

- thorough peer review by experienced researchers in your field

- rapid publication on acceptance

- support for research data, including large and complex data types

- gold Open Access which fosters wider collaboration and increased citations

- maximum visibility for your research: over $100 \mathrm{M}$ website views per year

At $\mathrm{BMC}$, research is always in progress.

Learn more biomedcentral.com/submissions 